ACADEMIA ROMÂNĂ

Revue Roumaine de Chimie

http://web.icf.ro/rrch/
Rev. Roum. Chim.

2020, 65(7-8), 699-705

DOI: $10.33224 /$ rrch.2020.65.7-8.07

\title{
SELECTIVE HYDRATION OF ELECTRON-RICH ARYL-ALKYNES BY A SCHROCK-TYPE MOLYBDENUM ALKYLIDENE CATALYST ${ }^{* *}$
}

\author{
Andreea CRIŞAN, ${ }^{\mathrm{a}}$ Alexandra POP,${ }^{\mathrm{a}}$ Elena BOGDAN, ${ }^{\mathrm{a}}$ Mihaela MATACHE,${ }^{\mathrm{b}}$ Lidia POP, \\ Anamaria TEREC, ${ }^{a}$ Ion GROSU ${ }^{\mathrm{a},{ }^{*}}$ and Niculina HĂDADE ${ }^{\mathrm{a}, *}$ \\ ${ }^{a}$ Babeș-Bolyai University, Faculty of Chemistry and Chemical Engineering, Supramolecular Organic \\ and Organometallic Chemistry Centre, 11 Arany Janos Str., RO-400028-Cluj-Napoca, Roumania \\ ${ }^{b}$ University of Bucharest, Faculty of Chemistry; Department of Organic Chemistry, Biochemistry and Catalysis, \\ Research Centre of Applied Organic Chemistry, 90-92 Panduri Street, RO-050663 Bucharest, Roumania
}

We present herein the regioselective hydration of electron-rich aryl-alkynes in presence of a Schrocktype molybdenum alkylidene catalyst. The structures of the obtained ketones were confirmed by NMR spectroscopy and HRMS as well as by single-crystal X-ray diffraction. We found out that in our

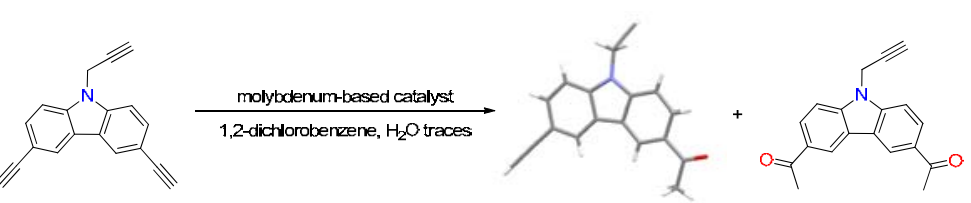
conditions the hydration reaction is efficient only for aryl-alkynes and their reactivity is highly dependent on the electronic nature of the substituents on the aryl group.

\section{INTRODUCTION}

The catalytic addition of water to alkynes, known as hydration, is a well-known reaction, introduced by Kucherov at the end of the $19^{\text {th }}$ century. ${ }^{1}$ Since then, this reaction was used to synthesise numerous carbonyl compounds with various applications, therefore, the alkyne group could be seen as a carbonyl equivalent.

A large number of catalysts have been reported to efficiently catalyze the alkynes hydration so far. The most encountered catalysts for hydration of terminal alkynes have been beyond doubt mercury (II) salts such as: mercury bromide, ${ }^{1}$ mercury sulphate, ${ }^{3}$ mercury acetate, ${ }^{4}$ mercury triflate ${ }^{5}$ etc., that yielded methyl ketones with Markovnikov selectivity. However, the toxicity of the mercury(II) catalysts prompted research to identify safer and more ecological catalysts. To date, there have been reported many metal salts or complexes useful as catalysts for this transformation. For example cerium(IV) sulphate ${ }^{6}$ and tungsten hexacarbonyl ${ }^{7}$ were proved to be efficient catalysts for this reaction. In addition, various ruthenium derivatives have been tested. Thus, ruthenium (III) chloride $^{8}$ or ruthenium(III) loaded ion-exchange resin $^{9}$ were found to promote the alkynes hydration with Markovnikov selectivity. However, ruthenium(II)-based catalysts were able to hydrate terminal alkynes both with Markovnikov $\{e . g$. $\left[\mathrm{Ru}\left(\eta^{5}\right.\right.$-indenyl $\left.) \mathrm{Cl}\left(\eta^{4} \mathrm{COD}\right)\right], \mathrm{COD}=1,5$-cyclooctadiene $)^{10}\left(\eta^{6}\right.$-arene $\left.) \mathrm{RuCl}\left(\mathrm{C}_{5} \mathrm{H}_{4} \mathrm{~N}-2-\mathrm{CH}=\mathrm{NR}\right) \mathrm{Cl}\right]^{+11}$ and

\footnotetext{
*Corresponding authors: nbogdan@chem.ubbcluj.ro or igrosu@chem.ubbcluj.ro

${ }^{* *}$ Supplementary information on http://web.icf.ro/rrch/ or http://revroum.lew.ro
} 
anti- Markovnikov (e.g. $\left[\mathrm{RuCl}_{2}\left(\mathrm{C}_{6} \mathrm{H}_{6}\right)\left[\mathrm{PPh}_{2}\left(\mathrm{C}_{6} \mathrm{~F}_{5}\right)\right]^{12}\right.$ selectivity. Furthermore, rhodium(III) chloride in presence of quaternary ammonium salts ${ }^{13}$ and iridium(I) complexes ${ }^{14}$ also provided Markovnikov products. Palladium(II) ${ }^{15}$ and platinum(IV $)^{16}$ as well as copper(I) and copper(II) ${ }^{17}$ have been also reported as efficient catalysts for this reaction.

The last decades witnessed a great interest for use of gold-based compounds as catalysts for alkynes hydration. The first reported example was $\mathrm{HAuCl}_{4}{ }^{18}$ Since then, other highly efficient cationic gold(I) systems of type $\left[\mathrm{L}-\mathrm{Au}^{+}\right]$ $(\mathrm{L}=\text { phosphane, arsane or phosphate }]^{19}$ or gold(I) $\mathrm{N}$-Heterocyclic Carbene complexes $(\mathrm{NHC})^{20}$ have been reported.

In this context, we report herein the molybdenum-mediated hydration of terminal or internal electron-rich aryl-alkynes yielding the corresponding carbonyl compounds. To the best of our knowledge this is the first example of alkynes hydration catalysed by a molybdenum-based catalyst.

\section{RESULTS AND DISCUSSION}

In our attempt to perform alkynes metathesis reaction between the trialkyne 4 in Scheme 1 and 1,4-di(prop-1-yn-1-yl)benzene we serendipitously discovered that the molybdenum catalyst I (Figure 1) catalyzed hydration of the triple bonds grafted on the aromatic rings of $\mathbf{4}$ while the $N$-2-butyn-1yl group was not affected. In addition, the di(prop-1yn-1-yl)benzene remained unaffected.<smiles>CCCc1cccc(C(C)C)c1N=C=NC(C)(C)c1ccccc1</smiles>

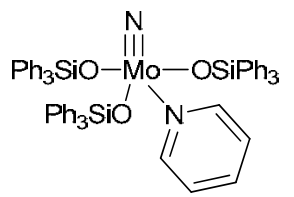

Fig. 1 - Structure of the molybdenum-based catalysts used in this work.

The trialkyne 4 was obtained in four steps (Scheme 1). The Sonogashira reaction between 3,6-diiodo-9H-carbazole and trimethylsilylacetylene followed by deprotection of trimethylsilyl groups allowed the synthesis of the diethynyl-decorated derivative $\mathbf{2}$ in near quantitative yield. Next, $\mathrm{N}$-propargyl group was introduced by reaction of compound $\mathbf{2}$ with propargyl bromide in basic medium and the three triple bonds in the structure of $\mathbf{3}$ were methylated using $n$-BuLi and methyl iodine.

Treatment of compound 4 with 1,4-di(prop-1yn-1-yl)benzene, in presence of catalyst I 5 mol\% in 1,2-dichlorobenzene dried over $\mathrm{CaCl}_{2}$, and distilled from $\mathrm{CaH}_{2}$ led to a mixture of mono- and diketones $\mathbf{5}$ and $\mathbf{6}$, respectively (Scheme 2) as a result of the regioselective addition of water (traces, ppm level in the solvent) to the triple bonds grafted on the aromatic rings. A similar result was obtained when the reaction was carried out in toluene (dried over sodium in presence of benzophenone). The same reaction performed in presence of molybdenum catalyst II (Figure 1) led to full recovery of the starting materials.

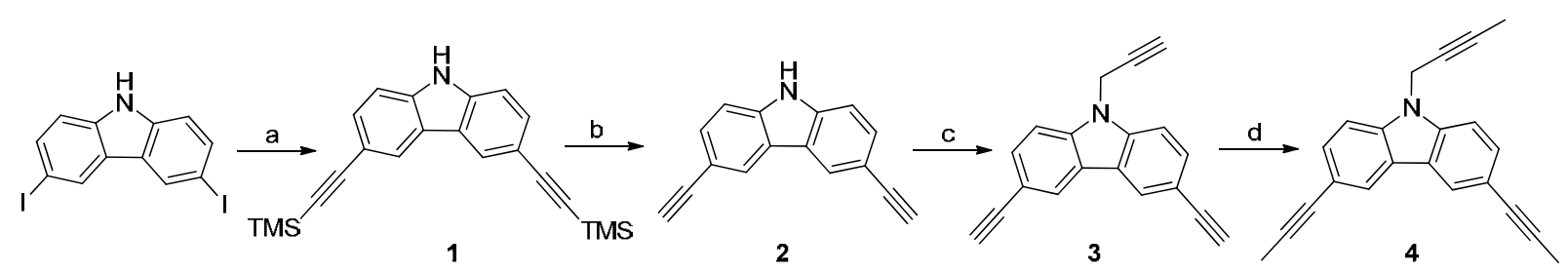

Scheme 1 - Synthesis of trialkyne 4. Reagents and conditions: a) trimethylsilylacetylene, $\mathrm{Pd}\left(\mathrm{PPh}_{3}\right)_{2} \mathrm{Cl}_{2}, \mathrm{PPh}_{3}, \mathrm{CuI}_{2} \mathrm{Et}_{3} \mathrm{~N}$, reflux, $97 \%$; b) $\mathrm{K}_{2} \mathrm{CO}_{3}$, $\mathrm{MeOH}$, r.t., $98 \%$; c) propargyl bromide, $\mathrm{KOH}$, DMF, r.t. $81 \%$; d) $n$-BuLi, MeI, THF, $-78{ }^{\circ} \mathrm{C}, 55 \%$.

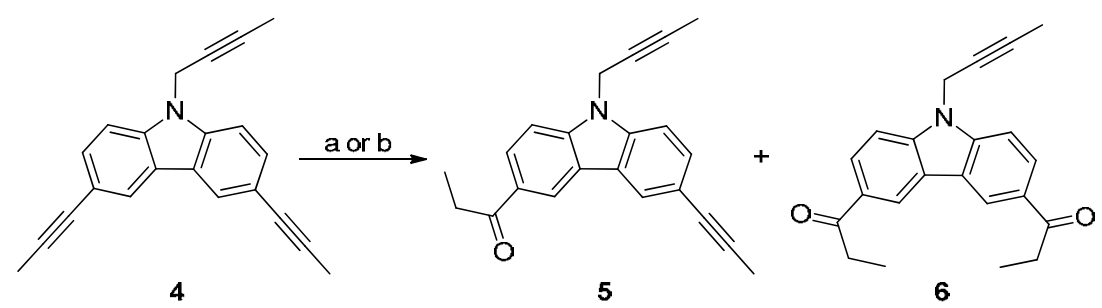

Scheme 2 - Hydration of compound 4 in presence of the molybdenum-based catalyst I. Reagents and conditions: a) 1,4-di(prop-1-yn-1-yl)benzene, catalyst I, 1,2-dichlorobenzene, $140{ }^{\circ} \mathrm{C}, 71 \% \mathbf{5}, 23 \% \mathbf{6}$; b) 1,4-di(prop-1-yn-1-yl)benzene, catalyst I, toluene, $110^{\circ} \mathrm{C}, 54 \% \mathbf{5}, 15 \% \mathbf{6}$ 


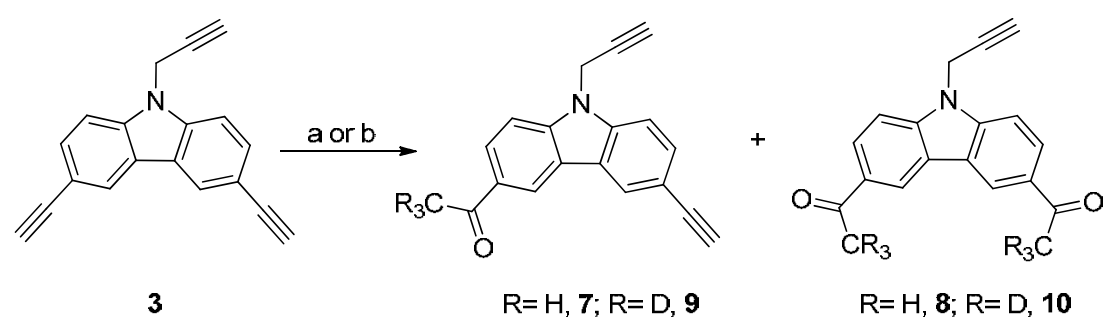

Scheme 3 - Hydration of compound $\mathbf{3}$ in presence of molybdenum-based catalyst $\mathbf{I}$. Reagents and conditions: a) catalyst I, 1,2dichlorobenzene, $140{ }^{\circ} \mathrm{C}, 53 \% 7,13 \% \mathbf{8}$; b) $\mathrm{D}_{2} \mathrm{O}$, catalyst I, 1,2-dichlorobenzene, $140{ }^{\circ} \mathrm{C}, 3 \% \mathbf{9}, 40 \% 10$.

To gain more insight on this hydration reaction, we treated compound $\mathbf{3}$ with catalyst $\mathbf{I}$ in anhydrous 1,2-dichlorobenzene at $140{ }^{\circ} \mathrm{C}$. The reaction yielded a mixture of mono- and di- $\alpha-$ methyl ketones $7(53 \%)$ and 8 (13\%) with Markovnikov selectivity (Scheme 3). When the same reaction was performed in presence of deuterated water the corresponding deuteratedmethyl ketones 9 (3\%) and 10 (40\%) were obtained. Thus, addition of the deuterated water led to increase in the yield of the diketone and decrease in the yield of the monoketone. In all reactions, the $\mathrm{N}$-2-butyn-1yl group was preserved.

Noteworthy, heating compound $\mathbf{3}$ in 1,2dichlorobenzene at $140{ }^{\circ} \mathrm{C}$, in presence of deuterated water, and in the absence of catalyst I did not affected any of the triple bonds in the structure of 3 .

Formation of ketones 5-10 and the selectivity of the reaction were proved by NMR spectroscopy and HRMS. In addition, crystallization of compound 7 from a diluted solution in chloroform, by slow evaporation, afforded single crystal suitable for X-ray diffraction (Figure 2).

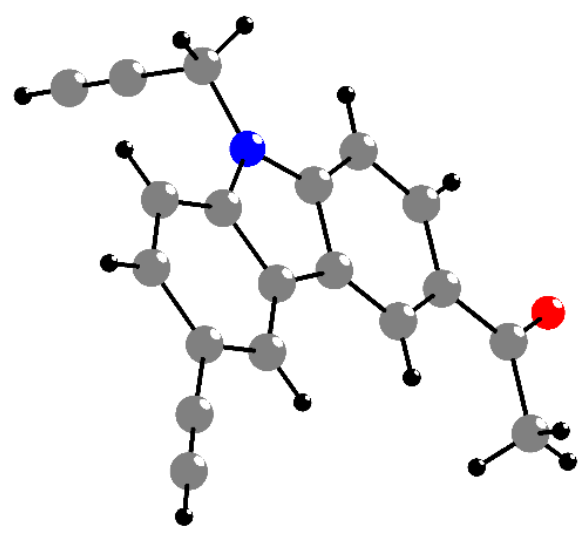

Fig. 2 - X-ray single-crystal molecular structure of the mono-ketone 7 .

Thus, the molecular structure of 7 confirms the Markovnikov selectivity of the hydration reactions and displayed the expected bond length and angle. The carbazole core is almost planar, the angle between the planes of the phenyl rings is $2.4^{\circ}$ and the substituents at positions 3 and 6 are almost orthogonal (i.e. the angle between $\underline{\mathrm{C}} \equiv \mathrm{CH}-\underline{\mathrm{N}}-\underline{\mathrm{C}}=\mathrm{O}$ is $93.1^{\circ}$.

Crystal packing of compound 7, viewed along c axis, consists of supramolecular chains of stacked molecules in a zig-zag arrangement (Figure 3). Each chain is built up by molecules of 7 having head to tail orientations and hold together by $\pi-\pi$ interactions between the ethynyl-decorated phenyl rings, thus forming dimers. These molecules were quasi-parallel and the distance between these planes about $3.590 \AA$. For each dimer unit there are two $\mathrm{C}-\mathrm{H}---\mathrm{O}$ contacts $(\mathrm{C}=\mathrm{O}$ group and $\mathrm{H}$ atoms in propargyl position) with dimers of the same chain and two similar contacts with dimers of the neighbour chain $(\mathrm{C}-\mathrm{H}---\mathrm{O}=2.588 \AA)$. In addition, the dimers belonging to neighbor chains display double (direct at inverted) $\mathrm{C}-\mathrm{H}-\pi$ interactions (C$\mathrm{H}---\pi=2.889 \AA$ ) between the $\mathrm{H}$ atom at position 2 of the carbazole units and the $\mathrm{N}$ connected propargyl group, thus delimiting new dimeric (macrocyclic) entities.

A similar result was obtained when compound 13 containing the ethynyl groups at position 2 and 7 of the carbazole core (Scheme 4), was submitted to the hydration reaction using $\mathbf{I}$ as catalyst. Compound $\mathbf{1 3}$ was obtained in three steps starting from 2,7-dibromo-9H-carbazole, using a similar strategy as for the synthesis of compound $\mathbf{3}$ (Scheme 4). However, the attempt to hydrate 2,7diethynyl-9H-fluoren-9-one using $\mathbf{I}$ as catalyst, in the same condition as above, did not yielded the expected ketones and we only recovered untransformed starting materials.

Thus, catalyst I efficiently hydrated terminal or internal triple bonds grafted on aromatic rings functionalised with electron donating substituents as in the case of compounds 3, 4 and 13 but was ineffective for hydration of $N$-2-butyn-1-yl group contained in the structure of these compounds or for the hydration of triple bonded grafted on aromatic rings that did not contain other substituents, as in the case of 1,4-di(prop-1-yn-1-yl)benzene or containing electron withdrawing substituents (e.g. 2,7-diethynyl9H-fluoren-9-one). 


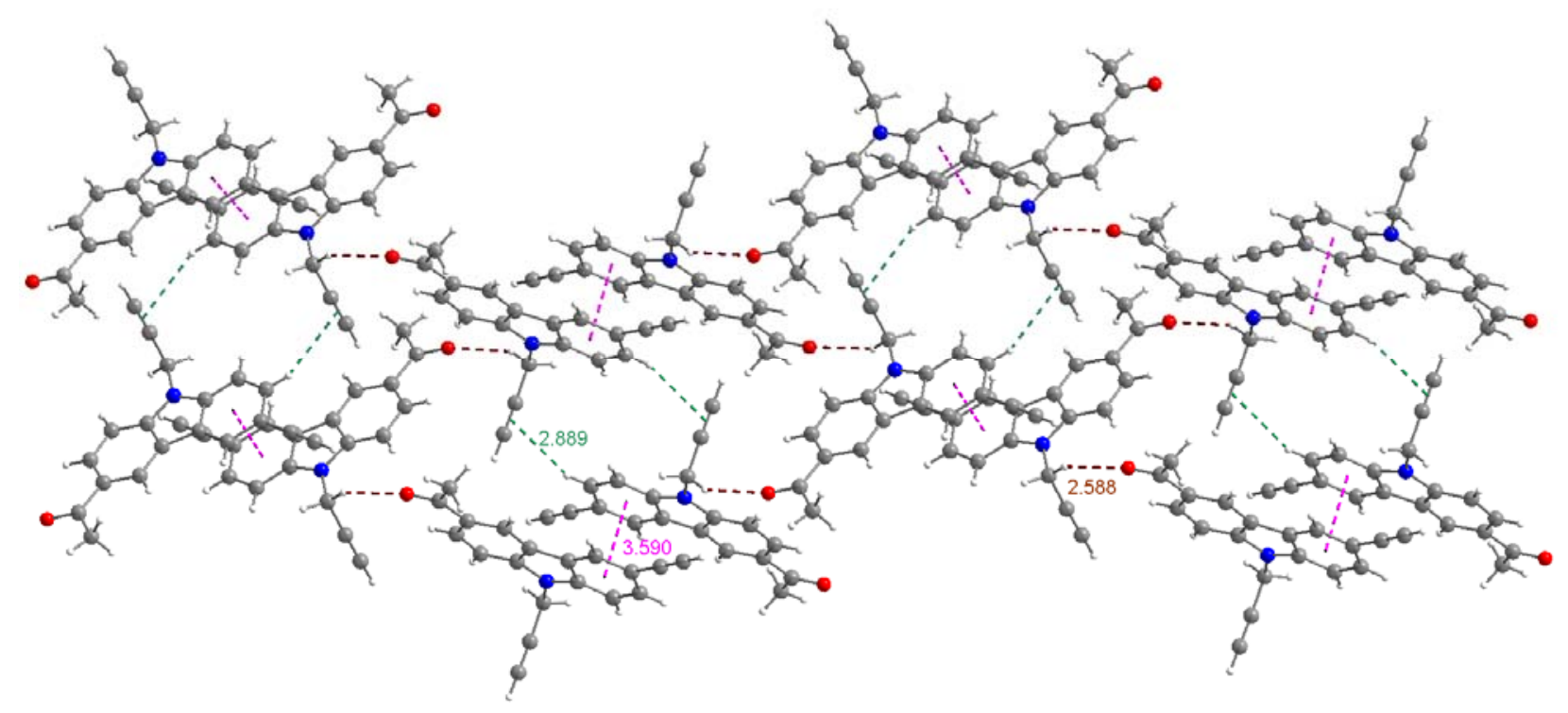

Fig. 3 - Crystal packing of compound 7 - view along $c$ axis.<smiles>C#Cc1ccc2cc(C#CCC)ccc2c1</smiles><smiles>[C-]#Cc1ccc2c3ccc(C(C)=O)cc3n(CC#C)c2c1</smiles>

Scheme 4 - Synthesis of trialkyne $\mathbf{1 3}$ and its hydration in presence of the molybdenum-based catalyst $\mathbf{I}$. Reagents and conditions: a) trimethylsilylacetylene, $\mathrm{Pd}\left(\mathrm{PPh}_{3}\right)_{2} \mathrm{Cl}_{2}, \mathrm{PPh}_{3}, \mathrm{CuI}, \mathrm{Et}_{3} \mathrm{~N}$, reflux, $82 \%$; b) $\mathrm{K}_{2} \mathrm{CO}_{3}, \mathrm{MeOH}$, r.t., $93 \%$; c) propargyl bromide, $\mathrm{KOH}$, DMF, r.t. $55 \%$; d) $\mathrm{H}_{2} \mathrm{O}$, catalyst I, 1,2-dichlorobenzene, $140{ }^{\circ} \mathrm{C}, 20 \% \mathbf{1 4}, 56 \% 15$.

\section{EXPERIMENTAL}

General experimental information. All reagents were purchased from commercial suppliers and used without further purification. Solvents were dried using standard procedures. Thin layer chromatography (TLC) was performed on silica gel 60 coated aluminium $F_{254}$ plates with visualisation by UV irradiation at $254 \mathrm{~nm}$. Preparative column chromatography was carried out using Merck silica gel 60, 0.040-0.063 mm. The NMR spectra were recorded on Bruker spectrometers operating at 600 or $400 \mathrm{MHz}$ for ${ }^{1} \mathrm{H}$ and 150 or $100 \mathrm{MHz}$ for ${ }^{13} \mathrm{C}$, respectively. Chemical shifts $(\delta)$ are reported in parts per million (ppm) using residual solvent peak as internal reference. High resolution mass spectra were recorded on a Thermo Scientific (LTQ XL Orbitrap) spectrometer, in positive ion mode, using APCI technique. Melting points were measured with a Kleinfeld melting point apparatus and are uncorrected.

The details of the crystal structure determination and refinement for compound 7 are given in Table S1. Data were collected on a Bruker SMART APEX diffractometer by using graphite-monochromated Mo-K $\alpha$ radiation $(\lambda=0.71073 \AA)$. The crystal was attached with epoxy glue on cryoloops and the data were collected at room temperature $(297 \mathrm{~K})$. The structure was refined with anisotropic thermal parameters. The hydrogen atoms were refined with a riding model and a mutual isotropic thermal parameter. For structure solving and refinement the software package SHELX-2014 was used. ${ }^{21}$ The drawings were created with Diamond program. ${ }^{22}$

3,6-Bis((trimethylsilyl)ethynyl)-9H-carbazole (1). In a flame-dried $250 \mathrm{~mL}$ round-bottom flask 3,6-diiodocarbazole $(3.0 \mathrm{~g}, 7.16 \mathrm{mmol})$ and trimethylsilylacetylene $(4.7 \mathrm{~mL}$, $32.9 \mathrm{mmol})$ were dissolved in dry triethylamine $(100 \mathrm{~mL})$. A mixture of $\left[\left(\mathrm{PPh}_{3}\right)_{2} \mathrm{PdCl}_{2}\right] \quad(226 \mathrm{mg}, 0.32 \mathrm{mmol})$, triphenylphosphine ( $43 \mathrm{mg}, 0.16 \mathrm{mmol}$ ), and $\mathrm{CuI}$ (40 mg, 0.2 mmol) was added, and the suspension was refluxed for $12 \mathrm{~h}$ under stirring and argon atmosphere. After removal of the volatile solvents, the crude product was solubilised in dichloromethane and filtered off through a pad of Celite. The filtrate was evaporated under reduced pressure and the resulting residue was separated by column chromatography (silica gel) using pentane/diethyl ether $3 / 2 \mathrm{v} / \mathrm{v}$ as eluent. Yellow solid. Yield: $97 \%$. M.p.: $212-214{ }^{\circ} \mathrm{C} . \mathrm{R}_{\mathrm{f}}=0.55$ (silica gel, pentane/diethyl ether $=3 / 2) .{ }^{1} \mathbf{H}$ NMR $(600 \mathrm{MHz}$, $\left.\mathrm{CDCl}_{3}\right), \delta_{\mathrm{H}}(\mathrm{ppm}): 0.29(\mathrm{~s}, 18 \mathrm{H}), 7.33(\mathrm{~d}, J=8.4 \mathrm{~Hz}, 2 \mathrm{H})$, $7.54\left(\mathrm{dd}, J=8.4, J^{\prime}=1.4 \mathrm{~Hz}, 2 \mathrm{H}\right), 8.16(\mathrm{~s}, 1 \mathrm{H}), 8.18(\mathrm{~d}$, $J=1.4 \mathrm{~Hz}, 2 \mathrm{H}) .{ }^{13} \mathbf{C} \mathbf{N M R}\left(150 \mathrm{MHz}, \mathrm{CDCl}_{3}\right), \delta_{\mathrm{C}}(\mathrm{ppm}): 0.3$, 
92.3, 106.3, 110.8, 114.7, 123.0, 124.8, 130.4, 139.6. HRMS (APCI+): $\mathrm{m} / z$ calcd. for $\mathrm{C}_{22} \mathrm{H}_{25} \mathrm{NSi}_{2}[\mathrm{M}]^{+}: 359.1520$, found: 359.1540 .

3,6-Diethynyl-9H-carbazole (2) To a solution of 1 (2.5 g, $6.9 \mathrm{mmol})$ in a mixture of methanol/dichloromethane $(100 \mathrm{~mL}$, $4 / 1 \mathrm{v} / \mathrm{v})$ anhydrous $\mathrm{K}_{2} \mathrm{CO}_{3}(1.9 \mathrm{~g}, 13.9 \mathrm{mmol})$ was added. The reaction mixture was stirred at room temperature for $48 \mathrm{~h}$ followed by complete removal of the solvent. The obtained residue was dissolved in dichloromethane, washed with water and brine, dried over magnesium sulphate, filtered off and evaporated in vacuo. No further purification was necessary. White solid. Yield: 98 \%. M.p.: 194-196 ${ }^{\circ} \mathrm{C} .{ }^{1} \mathbf{H}$ NMR $\left(600 \mathrm{MHz}, \mathrm{CDCl}_{3}\right), \delta_{\mathrm{H}}(\mathrm{ppm}): 3.08(\mathrm{~s}, 2 \mathrm{H}), 7.37$ (d, $J=8.3 \mathrm{~Hz}$, $2 \mathrm{H}), 7.57\left(\mathrm{dd}, J=8.3, J^{\prime}=1.4 \mathrm{~Hz}, 2 \mathrm{H}\right), 8.20(\mathrm{~d}, J=1.4 \mathrm{~Hz}, 2 \mathrm{H})$. ${ }^{13}$ C NMR (150 MHz, $\left.\mathrm{CDCl}_{3}\right), \delta_{\mathrm{C}}$ (ppm): 92.3, 106.3, 110.8, 114.6, 122.9, 124.8, 130.4, 139.6. HRMS (APCI+): $\mathrm{m} / \mathrm{z}$ calcd. for $\mathrm{C}_{16} \mathrm{H}_{10} \mathrm{~N}[\mathrm{M}+\mathrm{H}]^{+}: 216.0808$, found: 216.0813 .

3,6-diethynyl-9-(prop-2-yn-1-yl)-9H-carbazole (3). 3,6diethynyl-9H-carbazole $2(1.3 \mathrm{~g}, 6.08 \mathrm{mmol})$ and potassium hydroxide $(673 \mathrm{mg}, 12 \mathrm{mmol})$ were dissolved in dry DMF $(5 \mathrm{~mL})$ under argon and stirred for $10 \mathrm{~min}$ at room temperature. Propargyl bromide $(0.7 \mathrm{~mL}, 7.9 \mathrm{mmol})$ was added and the reaction mixture was stirred under inert atmosphere for $16 \mathrm{~h}$. The reaction mixture was poured into icewater and extracted three times with ethyl acetate. The organic layer was dried over magnesium sulphate, evaporated under reduced pressure and the resulting residue was separated by column chromatography (silica gel) using pentane/diethyl ether $(5 / 1 \mathrm{v} / \mathrm{v})$ as eluent. White solid. Yield: $81 \%$. M.p. $=194$ $196{ }^{\circ} \mathrm{C} . \mathrm{R}_{\mathrm{f}}=0.4$ (silica, Pentane/Diethyl ether $=5 / 1$ ). ${ }^{1} \mathbf{H}$ NMR (600 MHz, $\left.\mathrm{CDCl}_{3}\right), \delta_{\mathrm{H}}(\mathrm{ppm}): 2.30$ (t, $\left.J=2.5 \mathrm{~Hz}, 1 \mathrm{H}\right)$, $3.09(\mathrm{~s}, 2 \mathrm{H}), 5.03(\mathrm{~d}, J=2.5 \mathrm{~Hz}, 2 \mathrm{H}), 7.43(\mathrm{~d}, J=8.5 \mathrm{~Hz}, 2 \mathrm{H})$, $7.64\left(\mathrm{dd}, J=8.5, J^{\prime}=1.4 \mathrm{~Hz}, 1 \mathrm{H}\right), 8.22(\mathrm{~d}, J=1.4 \mathrm{~Hz}, 2 \mathrm{H})$. ${ }^{13} \mathrm{C}$ NMR $\left(150 \mathrm{MHz}, \mathrm{CDCl}_{3}\right), \delta_{\mathrm{C}}(\mathrm{ppm}): 29.8,32.7,73.1$, 75.9, 84.6, 109.2, 113.8, 122.8, 125.0, 130.6, 140.2. HRMS (APCI+): $m / z$ calcd. for $\mathrm{C}_{19} \mathrm{H}_{12} \mathrm{~N}[\mathrm{M}+\mathrm{H}]^{+}: 254.0964$, found: 254.0975 .

\section{9-(but-2-yn-1-yl)-3,6-di(prop-1-yn-1-yl)-9H-carbazole} (4). $n$-Butyllithium (2.4 M in hexane, $2.95 \mathrm{~mL}, 7.09 \mathrm{mmol})$ was added to a solution of derivative $3(0.5 \mathrm{~g}, 1.97 \mathrm{mmol})$ in anhydrous THF $(50 \mathrm{~mL})$ at $-78{ }^{\circ} \mathrm{C}$, under argon. The mixture was stirred for $1 \mathrm{~h}$. Then, iodomethane $(0.37 \mathrm{~mL}, 5.9 \mathrm{mmol})$ was added dropwise and stirred for $15 \mathrm{~h}$ at room temperature. The reaction mixture was poured into water and extracted with dichloromethane. The organic layer was separated, dried over magnesium sulphate and evaporated in vacuo. The crude product was purified by column chromatography on silica gel using a mixture of pentane/diethyl ether $=20 / 1 \mathrm{v} / \mathrm{v}$ as eluent. White solid. Yield: $55 \%$. M.p.: $182-183{ }^{\circ} \mathrm{C} . \mathrm{R}_{\mathrm{f}}=0.6$ (silica, Pentane/Diethyl ether $=20 / 1 \mathrm{v} / \mathrm{v}) .{ }^{1} \mathbf{H}$ NMR $(600 \mathrm{MHz}$, $\left.\mathrm{CDCl}_{3}\right), \delta_{\mathrm{H}}(\mathrm{ppm}): 1.75(\mathrm{t}, J=2.4 \mathrm{~Hz}, 3 \mathrm{H}), 2.11(\mathrm{~s}, 6 \mathrm{H}), 4.93$ (q, $J=2.4 \mathrm{~Hz}, 2 \mathrm{H}), 7.37$ (d, $J=8.4 \mathrm{~Hz}, 2 \mathrm{H}), 7.52(\mathrm{dd}, J=8.4 \mathrm{~Hz}$, $\left.J^{\prime}=1.2 \mathrm{~Hz}, 2 \mathrm{H}\right), 8.08(\mathrm{~d}, J=1.2 \mathrm{~Hz}, 2 \mathrm{H}) .{ }^{13} \mathbf{C} \mathbf{~ N M R}(100$ $\mathrm{MHz}, \mathrm{CDCl}_{3}$ ), $\delta_{\mathrm{C}}$ (ppm): 3.6, 4.6, 33.0, 72.9, 80.6, 80.7, 83.9, 109.1 115.2, 122.8, 123.9, 129.8, 139.6. HRMS (APCI+): $m / z$ calcd. for $\mathrm{C}_{22} \mathrm{H}_{17} \mathrm{~N}[\mathrm{M}+\mathrm{H}]^{+}: 296.1434$, found: 296.1452 .

1-(9-(But-2-yn-1-yl)-6-(prop-1-yn-1-yl)-9H-carbazol-3yl)propan-1-one (5) and 1,1'-(9-(but-2-yn-1-yl)-9Hcarbazole-3,6-diyl)bis(propan-1-one) (6). In a flame-dried 50 $\mathrm{mL}$ round-bottom Schlenk flask, 9-(but-2-yn-1-yl)-3,6di(prop-1-yn-1-yl)-9H-carbazole (4) (50 mg, $0.17 \mathrm{mmol}), 1,4-$ di(prop-1-yn-1-yl)benzene (39 mg, $0.25 \mathrm{mmol}$ ) and molybdenum catalyst I (13 mg, $10 \mathrm{~mol} \%)$ were dissolved in $12 \mathrm{~mL}$ anhydrous 1,2-dichlorobenzene. The mixture was heated at $140{ }^{\circ} \mathrm{C}$ for $48 \mathrm{~h}$, under argon. After removal of the solvent, the crude product was solubilised in dichloromethane and filtered through a pad of Celite. The filtrate was evaporated under reduced pressure and the resulting residue was purified by column chromatography (silica gel) using pentane/diethyl ether $(5 / 1, v / v)$ as eluent.

1-(9-(but-2-yn-1-yl)-6-(prop-1-yn-1-yl)-9H-carbazol-3yl)propan-1-one (5). White solid. Yield: $71 \%$. M.p.: 170-171 ${ }^{\circ} \mathrm{C} . \mathrm{R}_{\mathrm{f}}=0.42$ (silica, pentane/diethyl ether $=4 / 1$ ). ${ }^{1} \mathbf{H}$ NMR $\left(600 \mathrm{MHz}, \mathrm{CDCl}_{3}\right), \delta_{\mathrm{H}}(\mathrm{ppm}): 1.30(\mathrm{t}, J=7.2 \mathrm{~Hz}, 3 \mathrm{H}), 1.76(\mathrm{t}$, $J=2.4 \mathrm{~Hz}, 3 \mathrm{H}), 2.10(\mathrm{~s}, 3 \mathrm{H}), 3.13(\mathrm{q}, J=7.2 \mathrm{~Hz}, 2 \mathrm{H}), 4.99(\mathrm{q}$, $J=2.4 \mathrm{~Hz}, 2 \mathrm{H}), 7.43(\mathrm{~d}, J=8.4 \mathrm{~Hz}, 1 \mathrm{H}), 7.49(\mathrm{~d}, J=8.4 \mathrm{~Hz}$, $1 \mathrm{H}), 7.56$ (dd, $\left.J=8.4 \mathrm{~Hz}, J^{\prime}=1.2 \mathrm{~Hz}, 1 \mathrm{H}\right), 8.16(\mathrm{dd}, J=8.4 \mathrm{~Hz}$, $\left.J^{\prime}=1.8 \mathrm{~Hz}, 1 \mathrm{H}\right), 8.20(\mathrm{~d}, J=1.2 \mathrm{~Hz}, 1 \mathrm{H}), 8.7(\mathrm{~d}, J=1.2 \mathrm{~Hz}$, 1H). ${ }^{13} \mathbf{C}$ NMR $\left(150 \mathrm{MHz}, \mathrm{CDCl}_{3}\right), \delta_{\mathrm{C}}(\mathrm{ppm}): 3.6,4.6,8.9$, 31.8, 33.2, 72.5, 80.3, 81.0, 84.4, 108.9, 109.4, 116.1, 121.6, $122.7,123.5,124.1,126.7,129.4,130.2,139.9,143.0,200.4$. HRMS (APCI+): $m / z$ calcd. for $\mathrm{C}_{22} \mathrm{H}_{20} \mathrm{NO} \quad[\mathrm{M}+\mathrm{H}]^{+}$: 314.1539, found: 314.1536 .

1,1'-(9-(but-2-yn-1-yl)-9H-carbazole-3,6-diyl)bis(propan1-one) (6). Yellowish solid. Yield: $23 \%$. M.p.: $164-166{ }^{\circ} \mathrm{C}$. $\mathrm{R}_{\mathrm{f}}=0.2$ (silica, pentane/diethyl ether $\left.=4 / 1\right) .{ }^{1} \mathbf{H}$ NMR $(600$ $\left.\mathrm{MHz}, \mathrm{CDCl}_{3}\right), \delta_{\mathrm{H}}(\mathrm{ppm}): 1.31(\mathrm{t}, J=7.4 \mathrm{~Hz}, 6 \mathrm{H}), 1.77(\mathrm{t}, J=$ $2.4 \mathrm{~Hz}, 3 \mathrm{H}), 3.17$ (q, $J=7.4 \mathrm{~Hz}, 4 \mathrm{H}), 5.04$ (q, $J=2.4 \mathrm{~Hz}, 2 \mathrm{H})$, $7.55(\mathrm{~d}, J=8.4 \mathrm{~Hz}, 2 \mathrm{H}), 8.21\left(\mathrm{dd}, J=8.4 \mathrm{~Hz}, J^{\prime}=1.2 \mathrm{~Hz}\right.$, $2 \mathrm{H}), 8.80(\mathrm{~d}, J=1.2 \mathrm{~Hz}, 2 \mathrm{H}) .{ }^{13} \mathbf{C}$ NMR $\left(150 \mathrm{MHz}, \mathrm{CDCl}_{3}\right)$, $\delta_{\mathrm{C}}(\mathrm{ppm}): 3.6,8.8,31.9,33.4,72.2,81.4,109.3,121.6,123.4$, 127.1, 130.0, 143.4, 200.4. HRMS (APCI+): $\mathrm{m} / \mathrm{z}$ calcd. for $\mathrm{C}_{22} \mathrm{H}_{22} \mathrm{NO}_{2}[\mathrm{M}+\mathrm{H}]^{+}: 332.1645$, found: 332.1642 .

\section{1-(6-ethynyl-9-(prop-2-yn-1-yl)-9H-carbazol-3-} yl)ethanone (7) and 1,1'-(9-(prop-2-yn-1-yl)-9H-carbazole3,6-diyl)diethanone (8). In a flame-dried $50 \mathrm{~mL}$ roundbottom Schlenk flask, 3,6-diethynyl-9-(prop-2-yn-1-yl)-9Hcarbazole (3) $(50 \mathrm{mg}, 0.2 \mathrm{mmol})$ and molybdenum catalyst I (15 $\mathrm{mg}, 10 \mathrm{~mol} \%$ ) were dissolved in anhydrous 1,2dichlorobenzene $(8 \mathrm{~mL})$. The mixture was heated at $140{ }^{\circ} \mathrm{C}$ for $48 \mathrm{~h}$ under argon. After removal of the solvent, the crude product was dissolved in dichloromethane and filtered through a pad of Celite. The filtrate was evaporated under reduced pressure and the resulting residue was purified by column chromatography (silica gel) using pentane/diethyl ether (3/2, $\mathrm{v} / \mathrm{v})$ as eluent.

1-(6-ethynyl-9-(prop-2-yn-1-yl)-9H-carbazol-3-

yl)ethanone (7). White solid. Yield: $53 \%$. $\mathrm{R}_{\mathrm{f}}=0.4$ (silica, pentane/diethyl ether $=3 / 2, \mathrm{v} / \mathrm{v}) .{ }^{1} \mathbf{H}$ NMR $(600 \mathrm{MHz}$, $\left.\mathrm{CDCl}_{3}\right), \delta_{\mathrm{H}}(\mathrm{ppm}): 2.32(\mathrm{t}, J=2.4 \mathrm{~Hz}, 1 \mathrm{H}), 2.73(\mathrm{~s}, 3 \mathrm{H}), 3.10$ $(\mathrm{s}, 1 \mathrm{H}), 5.06(\mathrm{~d}, J=2.4 \mathrm{~Hz}, 2 \mathrm{H}), 7.47(\mathrm{~d}, J=8.4 \mathrm{~Hz}, 1 \mathrm{H}), 7.52$ $(\mathrm{d}, J=8.4 \mathrm{~Hz}, 1 \mathrm{H}), 7.67\left(\mathrm{dd}, J=8.4 \mathrm{~Hz}, J^{\prime}=1.2 \mathrm{~Hz}, 1 \mathrm{H}\right)$, $8.18\left(\mathrm{dd}, J=8.4 \mathrm{~Hz}, J^{\prime}=2.4 \mathrm{~Hz}, 1 \mathrm{H}\right), 8.31(\mathrm{~d}, J=1.2 \mathrm{~Hz}$, $1 \mathrm{H}), 8.71(\mathrm{~d}, J=1.2 \mathrm{~Hz}, 1 \mathrm{H}) .{ }^{13} \mathbf{C}$ NMR $\left(150 \mathrm{MHz}, \mathrm{CDCl}_{3}\right)$, $\delta_{\mathrm{C}}(\mathrm{ppm}): 26.8,32.9,73.4,76.2,76.9,84.4,108.9,109.5$, $114.4,122.1,122.8,123.6,125.0,127.3,130.2,130.8,140.5$, 143.1, 197.5. HRMS (APCI+): $m / z$, calcd. for $\mathrm{C}_{19} \mathrm{H}_{14} \mathrm{NO}$ $[\mathrm{M}+\mathrm{H}]^{+}: 272.1070$, found: 272.1088 .

\section{1,1'-(9-(prop-2-yn-1-yl)-9H-carbazole-3,6-}

diyl)diethanone (8). Yellowish solid. Yield: $13 \% . \mathrm{R}_{\mathrm{f}}=0.13$ (silica, pentane/diethyl ether $=3 / 2, \mathrm{v} / \mathrm{v}) .{ }^{1} \mathbf{H}$ NMR $(600 \mathrm{MHz}$, $\left.\mathrm{CDCl}_{3}\right), \delta_{\mathrm{H}}(\mathrm{ppm}): 2.34(\mathrm{t}, J=2.4 \mathrm{~Hz}, 1 \mathrm{H}), 2.76(\mathrm{~s}, 6 \mathrm{H}), 5.11$ $(\mathrm{d}, J=2.4 \mathrm{~Hz}, 2 \mathrm{H}), 7.57$ (d, $J=8.4 \mathrm{~Hz}, 2 \mathrm{H}), 8.22(\mathrm{dd}, J=8.4 \mathrm{~Hz}$, 
$\left.J^{\prime}=1.2 \mathrm{~Hz}, 2 \mathrm{H}\right), 8.80(\mathrm{~d}, J=1.2 \mathrm{~Hz}, 2 \mathrm{H}) .{ }^{13} \mathbf{C} \mathbf{~ N M R}(150 \mathrm{MHz}$, $\left.\mathrm{CDCl}_{3}\right), \delta_{\mathrm{C}}(\mathrm{ppm}): 26.9,33.1,73.6,76.6,109.2,122.2,123.5$, 127.5, 130.6, 143.4, 197.5. HRMS (APCI+): $\mathrm{m} / \mathrm{z}$ calcd. for $\mathrm{C}_{19} \mathrm{H}_{16} \mathrm{NO}_{2}[\mathrm{M}+\mathrm{H}]^{+}: 290.1176$, found: 290.1182 .

Deuterated derivatives 9 and 10. To a degassed solution of 3,6-diethynyl-9-(prop-2-yn-1-yl)-9H-carbazole (3) (100 mg, $0.39 \mathrm{mmol})$ in dry 1,2-dichlorobenzene $(12 \mathrm{~mL})$ and deuterated water $(0.1 \mathrm{~mL})$, molybdenum catalyst I $(30 \mathrm{mg}, 10$ mol \%) was added. The reaction mixture was heated at $140{ }^{\circ} \mathrm{C}$ for $48 \mathrm{~h}$ under argon. After removal of the solvent, the crude product was dissolved in dichloromethane and filtered through a pad of Celite. The filtrate was evaporated under reduced pressure and the resulting residue was purified by column chromatography (silica gel) using pentane/diethyl ether (1/2 $\mathrm{v} / \mathrm{v}$ ) as eluent.

9. Yellowish solid. Yield: $3 \%$. M.p.: 204-205 ${ }^{\circ} \mathrm{C} . \mathrm{R}_{\mathrm{f}}=0.7$ (silica, pentane/diethyl ether $=1 / 2) .{ }^{1} \mathbf{H}$ NMR $(600 \mathrm{MHz}$, $\left.\mathrm{CDCl}_{3}\right), \delta_{\mathrm{H}}(\mathrm{ppm}): 2.32(\mathrm{t}, J=2.4 \mathrm{~Hz}, 0.3 \mathrm{H}), 2.69-2.73(\mathrm{~m}$, $1.3 \mathrm{H}), 3.1(\mathrm{~s}, 0.25 \mathrm{H}), 5.07(\mathrm{~s}, 2 \mathrm{H}), 7.47(\mathrm{~d}, J=8.4 \mathrm{~Hz}, 1 \mathrm{H})$, $7.52(\mathrm{~d}, J=8.4 \mathrm{~Hz}, 1 \mathrm{H}), 7.67\left(\mathrm{dd}, J=8.4 \mathrm{~Hz}, J^{\prime}=1.2 \mathrm{~Hz}\right.$, $1 \mathrm{H}), 8.31(\mathrm{~d}, J=1.2 \mathrm{~Hz}, 1 \mathrm{H}), 8.71(\mathrm{~d}, J=1.2 \mathrm{~Hz}, 1 \mathrm{H})$. HRMS (APCI+): $\mathrm{m} / z$ calcd. for $\mathrm{C}_{19} \mathrm{H}_{8} \mathrm{D}_{5} \mathrm{NO}[\mathrm{M}]^{+}: 276.1305$, found: 276.1318 .

10. Yellow solid. Yield: $40 \% . . R_{\mathrm{f}}=0.37$ (silica, pentane/ diethyl ether $=1 / 2 \mathrm{v} / \mathrm{v}) .{ }^{1} \mathbf{H}$ NMR $\left(600 \mathrm{MHz}, \mathrm{CDCl}_{3}\right), \delta_{\mathrm{H}}$ (ppm): 2.34 (t, $J=2.4 \mathrm{~Hz}, 0.22 \mathrm{H}), 2.71-2.75(\mathrm{~m}, 2.24 \mathrm{H}), 5.1$ $(\mathrm{s}, 2 \mathrm{H}), 7.55(d, J=8.4 \mathrm{~Hz}, 2 \mathrm{H}), 8.21\left(\mathrm{dd}, J=8.4 \mathrm{~Hz}, J^{\prime}=1.2\right.$ $\mathrm{Hz}, 2 \mathrm{H}), 8.78(\mathrm{~d}, J=1.2 \mathrm{~Hz}, 2 \mathrm{H}) .{ }^{13} \mathbf{C}$ NMR $(150 \mathrm{MHz}$, $\left.\mathrm{CDCl}_{3}\right), \delta_{\mathrm{C}}(\mathrm{ppm}): 33.0,73.6,109.2,122.1,123.5,127.5$, 130.6, 143.4, 197.6. HRMS (APCI+): $\mathrm{m} / \mathrm{z}$ calcd. for $\mathrm{C}_{19} \mathrm{H}_{9} \mathrm{D}_{6} \mathrm{NO}_{2}[\mathrm{M}]^{+}: 295.1474$, found: 295.1467.

2,7-Bis((trimethylsilyl)ethynyl)-9H-carbazole (11). In a flame-dried $250 \mathrm{~mL}$ round-bottom flask, 2,7-dibromocarbazole $(1.0 \mathrm{~g}, 3.08 \mathrm{mmol})$ and trimethylsilylacetylene $(2.0 \mathrm{~mL}$, $14.15 \mathrm{mmol})$ were dissolved in dry triethylamine $(40 \mathrm{~mL})$. A mixture of $\left[\left(\mathrm{PPh}_{3}\right)_{2} \mathrm{PdCl}_{2}\right] \quad\left(\begin{array}{llll}100 & \mathrm{mg}, & 0.14 & \mathrm{mmol}\end{array}\right)$, triphenylphosphine $(19 \mathrm{mg}, 0.07 \mathrm{mmol})$ and $\mathrm{CuI}(18 \mathrm{mg}, 0.09$ mmol) was added, and the suspension was refluxed for $12 \mathrm{~h}$ under stirring and argon. After removal of the volatile solvents, the crude product was solubilised in dichloromethane and filtered through a pad of Celite. The filtrate was evaporated under reduced pressure and the resulting residue was separated by column chromatography (silica gel) using pentane/diethyl ether $(9 / 1, \mathrm{v} / \mathrm{v})$ as eluent. Beige solid. Yield: $82 \%$. M.p.: $184-185{ }^{\circ} \mathrm{C} . \mathrm{R}_{\mathrm{f}}=0.46$ (silica, pentane/diethyl ether $=9 / 1, \mathrm{v} / \mathrm{v}) .{ }^{1} \mathbf{H}$ NMR $\left(600 \mathrm{MHz}, \mathrm{CDCl}_{3}\right), \delta_{\mathrm{H}}(\mathrm{ppm}): 0.28$ $(\mathrm{s}, 18 \mathrm{H}), 7.35\left(\mathrm{dd}, J=7.8 \mathrm{~Hz}, J^{\prime}=1.2 \mathrm{~Hz}, 2 \mathrm{H}\right), 7.54\left(\mathrm{~s}_{(\mathrm{br})}\right.$, $2 \mathrm{H}), 7.95(\mathrm{~d}, J=8.1 \mathrm{~Hz}, 2 \mathrm{H}), 8.04\left(\mathrm{~s}_{(\mathrm{br})}, 2 \mathrm{H}\right)$.

2,7-diethynyl-9H-carbazole (12) To a solution of derivative $11(0.9 \mathrm{~g}, 2.5 \mathrm{mmol})$ in a mixture of methanol/ dichloromethane $(50 \mathrm{~mL}, 4 / 1 \mathrm{v} / \mathrm{v})$, anhydrous $\mathrm{K}_{2} \mathrm{CO}_{3}(1.04 \mathrm{~g}$, $7.5 \mathrm{mmol}$ ) was added. The reaction mixture was stirred at room temperature for $48 \mathrm{~h}$ followed by complete removal of the solvent. The obtained residue was dissolved in dichloromethane, washed with water and brine, dried over magnesium sulfate, filtered off and evaporated in vacuo. No further purification was necessary. Yellowish solid. Yield: $93 \%$ \% ${ }^{1} \mathbf{H}$ NMR $\left(600 \mathrm{MHz}, \mathrm{CDCl}_{3}\right), \delta_{\mathrm{H}}(\mathrm{ppm}): 3.14$ (s, 2H), $7.38\left(\mathrm{dd}, J=7.8 \mathrm{~Hz}, J^{\prime}=1.2 \mathrm{~Hz}, 2 \mathrm{H}\right), 7.58(\mathrm{~s}, 2 \mathrm{H}), 7.99(\mathrm{~d}$, $J=8.4 \mathrm{~Hz}, 2 \mathrm{H}), 8.1(\mathrm{~s}, 1 \mathrm{H}) .{ }^{13} \mathbf{C}$ NMR $\left(150 \mathrm{MHz}, \mathrm{CDCl}_{3}\right), \delta_{\mathrm{C}}$ (ppm): 77.2, 84.7, 114.7, 119.8, 120.7, 123.5, 124.1, 139.7. HRMS (APCI+): $m / z$ calcd. for $\mathrm{C}_{16} \mathrm{H}_{10} \mathrm{~N}[\mathrm{M}+\mathrm{H}]^{+}: 216.0808$, found: 216.0805 .
2,7-diethynyl-9-(prop-2-yn-1-yl)-9H-carbazole (13). 2,7diethynyl-9H-carbazole $12(0.5 \mathrm{~g}, 2.3 \mathrm{mmol})$ and potassium hydroxide (260 mg, $4.6 \mathrm{mmol}$ ) were dissolved in dry DMF (3 $\mathrm{mL}$ ) under argon and stirred for $10 \mathrm{~min}$ at room temperature. Propargyl bromide $(0.27 \mathrm{~mL}, 3 \mathrm{mmol})$ was added and then the reaction mixture was stirred for $16 \mathrm{~h}$ under inert atmosphere. The reaction mixture was poured into ice-water and extracted three times with ethyl acetate. The organic layer was dried over magnesium sulphate, evaporated under reduced pressure and the resulting residue was separated by column chromatography (silica gel) using pentane/diethyl ether $(7 / 1$, $\mathrm{v} / \mathrm{v}$ ) as eluent. White solid. Yield: $55 \%$. M.p.: $144-145{ }^{\circ} \mathrm{C}$. $\mathrm{R}_{\mathrm{f}}=0.6$ (silica, pentane/diethyl ether $=7 / 1$ ). ${ }^{1} \mathbf{H}$ NMR $\left(600 \mathrm{MHz}, \mathrm{CDCl}_{3}\right), \delta_{\mathrm{H}}(\mathrm{ppm}): 2.30(\mathrm{t}, J=2.4 \mathrm{~Hz}, 1 \mathrm{H}), 3.17(\mathrm{~s}$, $2 \mathrm{H}), 5.00(\mathrm{~d}, J=2.4 \mathrm{~Hz}, 2 \mathrm{H}), 7.41\left(\mathrm{dd}, J=7.8 \mathrm{~Hz}, J^{\prime}=1.2\right.$ $\mathrm{Hz}, 2 \mathrm{H}), 7.64$ (s, 2H), 8.00 (d, $J=7.8 \mathrm{~Hz}, 2 \mathrm{H}) .{ }^{13} \mathbf{C}$ NMR $\left(150 \mathrm{MHz}, \mathrm{CDCl}_{3}\right), \delta_{\mathrm{C}}(\mathrm{ppm}): 32.6,73.1,77.2,77.4,84.7$, 113.0, 120.0, 120.8, 123.4, 124.2, 140.1. HRMS (APCI+): $\mathrm{m} / \mathrm{z}$ calcd. for $\mathrm{C}_{19} \mathrm{H}_{12} \mathrm{~N}[\mathrm{M}+\mathrm{H}]^{+}: 254.0964$, found: 254.0968 .

\section{1-(7-ethynyl-9-(prop-2-yn-1-yl)-9H-carbazol-2-}

yl)ethanone (14) and 1,1'-(9-(prop-2-yn-1-yl)-9H-carbazole2,7-diyl)diethanone (15). To a degassed solution of 2,7diethynyl-9-(prop-2-yn-1-yl)-9H-carbazole (13) (100 mg, $0.39 \mathrm{mmol})$ in dry 1,2-dichlorobenzene $(20 \mathrm{~mL})$ and water $(0.1 \mathrm{~mL})$, molybdenum catalyst I (30 $\mathrm{mg}, 10 \mathrm{~mol} \%)$ was added. The reaction mixture was heated at $140{ }^{\circ} \mathrm{C}$ for $48 \mathrm{~h}$ under argon. After removal of the solvent, the crude product was dissolved in dichloromethane and filtered through a pad of Celite. The filtrate was evaporated under reduced pressure and the resulting residue was purified by column chromatography (silica gel) using pentane/diethyl ether (3/2, $\mathrm{v} / \mathrm{v}$ ) as eluent.

1-(7-ethynyl-9-(prop-2-yn-1-yl)-9H-carbazol-2yl)ethanone (14). White solid. Yield: $20 \%$. M.p.: 229-231 ${ }^{\circ} \mathrm{C} . \mathrm{R}_{\mathrm{f}}=0.52$ (silica, pentane/diethyl ether $=3 / 2$ ). ${ }^{1} \mathbf{H}$ NMR $\left(400 \mathrm{MHz}, \mathrm{CDCl}_{3}\right), \delta_{\mathrm{H}}(\mathrm{ppm}): 2.31(\mathrm{t}, J=2.4 \mathrm{~Hz}, 1 \mathrm{H}), 2.75(\mathrm{~s}$, $3 \mathrm{H}), 3.20(\mathrm{~s}, 1 \mathrm{H}), 5.09(\mathrm{~d}, J=2.4 \mathrm{~Hz}, 2 \mathrm{H}), 7.43(\mathrm{dd}, J=8.0$ $\left.\mathrm{Hz}, J^{\prime}=1.2 \mathrm{~Hz}, 1 \mathrm{H}\right), 6.83(\mathrm{~s}, 1 \mathrm{H}), 7.89(\mathrm{dd}, J=8.0 \mathrm{~Hz}$, $\left.J^{\prime}=1.2 \mathrm{~Hz}, 1 \mathrm{H}\right), 8.07(\mathrm{~d}, J=8.0 \mathrm{~Hz}, 1 \mathrm{H}), 8.12(\mathrm{~d}, J=8.4 \mathrm{~Hz})$, $8.15(\mathrm{~s}, 1 \mathrm{H}) .{ }^{13} \mathbf{C}$ NMR $\left(100 \mathrm{MHz}, \mathrm{CDCl}_{3}\right), \delta_{\mathrm{C}}(\mathrm{ppm}): 27.2$, $32.7,73.2,77.8,84.5,109.1,113.2,120.7,120.8,120.9$, 121.4, 122.9, 124.4, 126.8, 135.4, 140.3, 141.0, 198.3. HRMS (APCI+): calc. for $\mathrm{C}_{19} \mathrm{H}_{14} \mathrm{NO}[\mathrm{M}+\mathrm{H}]^{+}:$272.1070, found: 272.1073 .

1,1'-(9-(prop-2-yn-1-yl)-9H-carbazole-2,7diyl)diethanone (15). Yellow solid. Yield: $56 \% \mathrm{R}_{\mathrm{f}}=0.2$ (silica, pentane/diethyl ether $=3 / 2)$. ${ }^{1} \mathbf{H}$ NMR $(600 \mathrm{MHz}$, $\left.\mathrm{CDCl}_{3}\right), \delta_{\mathrm{H}}(\mathrm{ppm}): 2.31(\mathrm{t}, J=2.4 \mathrm{~Hz}, 1 \mathrm{H}), 2.76(\mathrm{~s}, 6 \mathrm{H}), 3.20$ $(\mathrm{s}, 1 \mathrm{H}), 5.18(\mathrm{~d}, J=2.4 \mathrm{~Hz}, 2 \mathrm{H}), 7.92\left(\mathrm{dd}, J=8.4 \mathrm{~Hz}, J^{\prime}=1.8 \mathrm{~Hz}\right.$, 2H) , 8.18-8.20 (overlapped peaks, 4H) ${ }^{13} \mathbf{C}$ NMR (150 MHz, $\left.\mathrm{CDCl}_{3}\right), \delta_{\mathrm{C}}(\mathrm{ppm}): 27.2,32.8,73.4,109.4,120.7,121.3,126.4$, 136.1, 141.2, 198.2. HRMS (APCI+): calc. for $\mathrm{C}_{19} \mathrm{H}_{16} \mathrm{NO}_{2}$ $[\mathrm{M}+\mathrm{H}]^{+}: 290.1176$, found: 290.1176 .

Supporting Information Available: full spectroscopic data for all new compounds, details of the crystal structure determination and refinement for compound 7. CCDC 1959578 contains the supplementary crystallographic data for compound 7. These data can be obtained free of charge from The Cambridge Crystallographic Data Centre via www.ccdc.cam.ac.uk/data_request/cif. 


\section{CONCLUSIONS}

In summary, we have shown that the molybdenum-based catalyst $\mathbf{I}$ is able to regioselectively hydrate terminal or internal electron-rich arylalkynes. Structural characterization of the obtained ketones by NMR spectroscopy and X-ray diffraction was in agreement with the synthesis of the Markovnikov products. This catalyst could find important applications in organic synthesis for selective hydration of alkyne groups grafted on electron-rich aromatic rings in presence of other alkyne groups on unsubstituted or electron-poor aromatic rings as well as in the presence of aliphatic alkyne groups.

Acknowledgements. Financial support by Roumanian National Authority for Scientific Research and Innovation, CNCS-UEFISCDI, project number PN-III-P4-ID-PCCF-20160088 , is gratefully acknowledged.

\section{REFERENCES}

1. M. Kutscheroff, Ber. Dtsch. Chem. Ges., 1881, 14, 15401542.

2. (a) F. Alonso, I. P. Beletskaya and M. Yus, Chem. Rev., 2004, 104, 3079-3159; (b) W. E. Brenzovich, Angew. Chem. Int. Ed., 2012, 51, 8933-8935; (c) L. Hintermann, Top. Organomet. Chem., 2010, 31, 123-155; (d) B. Xu, W. Wang, L.-P. Liu, J. Han, Z. Jin and G. B. Hammond, J. Organomet. Chem., 2011, 696, 269-276; (e) L. Hintermann and A. Labonne, Synthesis, 2007, 8, 1121-1150.

3. (a) M. Kutscheroff, Chem. Ber., 1884, 17, 13-29; (b) R. J. Thomas, K. N. Campbell and G. F. Hennion, J. Am. Chem. Soc., 1938, 60, 718-720.

4. M. G. Kutscheroff, Chem. Ber. 1909, 42, 2759-2762.

5. M. Nishizawa, Hiroko Takao, V. K. Yadav, H. Imagawa and T. Sugihara, Org. Lett., 2003, 52, 4563-4565.
6. W. Liu and J. Li, Chin. J. Org. Chem., 2006, 26, 10731078.

7. B. Koo and F. E. McDonald, Org. Lett., 2005, 7, 3621-3627.

8. J. Halpern, B. R. James and A. L. W. Kemp, J. Am. Chem. Soc., 1966, 88, 5142-5147.

9. I. K. Meier and J. A. Marsella, J. Mol. Catal., 1993, 78, $31-42$.

10. P. Alvarez, J. Gimeno, E. Lastra, S. García-Granda, Van J. F. der Maelen and M. Bassetti, Organometallics, 2001, 20, 3762-3771.

11. D. Tyagi, R. K. Rai, S. M. Mobin and S. K. Singh, Asian J. Org. Chem., 2017, 6, 1647-1658.

12. M. Tokunaga and Y. Wakatsuki, Angew. Chem. Int. Ed., 1998, 37, 2867-2869.

13. J. Blum, H. Huminer and H. Alper, J. Mol. Catal., 1992, $75,153-160$.

14. C. S. Chin, W.-T. Chang, S. Yang and K.-S. Joo, Bull. Korean Chem. Soc., 1997, 18, 324-327.

15. (a) K. Yasutaka , M. Osamu, O. Masataka, Y. Tsuneaki and T. Kazuhide, Chem. Lett., 1994, 1283-1284; (b) A. Arcadi, S. Cacchi and F. Marinelli, Tetrahedron, 1993, 49, 4955-4964; (c) J. W. Hartman, W. C. Hiscox and P. W. Jennings, J. Org. Chem., 1993, 58, 7613-7614.

16. O. Israelsohn, K. P. C. Vollhardt and J. Blum, J. Mol. Catal., 2002, 184, 1-10.

17. Y. Tokita, A. Okamoto, K. Nishiwaki, M. Kobayashi and E. Nakamura, Bull. Chem. Soc. Jpn., 2004, 77, 1395-1399.

18. Y. Fukuda and K. Utimoto, J. Org. Chem., 1991, 56, 3729-3731.

19. (a) J. H. Teles, S. Brode and M. Chabanas, Angew. Chem. Int. Ed., 1998, 37, 1415-1418; (b) E. Mizushima, K. Sato, T. Hayashi and M. Tanaka, Angew. Chem. Int. Ed., 2002, 41, 4563-4565.

20. (a) N. Marion, R. S. Ramón and S. P. Nolan, J. Am. Chem. Soc., 2008, 131, 448-449; (b) H. Ibrahim, P. de Frémont, P. Braunstein, V. Théry, L. Nauton, F. Cisnetti and A. Gautier, Adv. Synth. Catal., 2015, 357, 3893 -3900; (c) M. Heidrich, M. Bergmann, D. Müller-Borges and H. Plenioa, Adv. Synth. Catal., 2018, 360, $3572-3578$.

21. G. M. Sheldrick, Acta Crystallogr. Sect. A, 2008, 64, 112-122.

22. DIAMOND - Visual Crystal Structure Information System, CRYSTAL IMPACT, Postfach 1251, 53002 Bonn, Germany, 2001. 
